\title{
TWO DIFFERENT STELLAR POPULATIONS IN THE LARGE
}

\section{MAGELLANIC CLOUD?}

\author{
JU. FRANTSMAN AND I. SHMELD \\ Radioastrophysical observatory, Latvian Acad. Sci. \\ Turgeneva 19, Riga, LV-1527 LATVIA
}

The evolution of stars on the AGB has been explained as episodic helium burning and neutron capture nucleosynthesis in the stellar interior, followed by the mixing of a portion of the processed material into the envelope of a star. The simple stellar evolutionary scheme M-MS-S-SC-N had been accepted. However in recent years extensive observations of AGB stars have shown that evolution of the AGB is more complicated than painted in such a simple picture.

AGB stars are grouped into two classes. Those which have not yet begun to thermal pulse (E-AGB), and those that are in the thermally pulsing phase (TP-AGB). The theory of two AGB stages (E-AGB and TP-AGB) was developed more than decade ago but some authors still do not take into account the E-AGB phase in spite of the fact that it lasts for some stars considerably longer than the TP-AGB phase. The typical outcome of such neglect is deduction of two episodes of star formation and two different stellar populations in the Large Magellanic Cloud.

One of the most serious argument for two distinct active epochs of star formation in the LMC is the colour-magnitude diagram for field and cluster cool luminous M giants. Two well-defined AGBs for these stars have been found and the authors came to the conclusion that this division is associated with star formation epochs a few Gyr ago and about $10^{8} \mathrm{yr}$ ago: the second epoch may still be in progress. We have explained these two regions of star concentration on the colour-magnitude diagram in a quite different manner, theoretically, taking into account two phases of AGB evolution (E-AGB and TP-AGB).

The effect of the E-AGB phase is very important in the problem of cluster age determination on the basis of a relation between age and maximum AGB luminosity. Before using the luminosity of AGB stars for the determination of age it is necessary to exclude E-AGB stars. 